\title{
Statistical relationship between CME speed and soft X-ray peak flux of the associated flare during solar cycle 23
}

\author{
C. Salas-Matamoros ${ }^{1}$, K.-L. Klein ${ }^{2}$ and L. Taliashvili ${ }^{1}$ \\ ${ }^{1}$ Space Research Center, University of Costa Rica (CINESPA) \\ email: carolina.salas@planetario.ucr.ac.cr \\ ${ }^{2}$ LESIA - UMR 8109, Observatoire de Paris, CNRS, Univ. Paris 6 \& 7 \\ email: ludwig.klein@obspm.fr
}

\begin{abstract}
The relationship between the speed of coronal mass ejections and the peak soft X-ray flux of the associated flares is studied for events occurring near the solar limbs between 1996 and 2008. An improved, though still moderate, correlation between the two parameters is found.
\end{abstract}

Keywords. Coronal mass ejections, flares, X-ray emission

\section{Introduction}

Coronal Mass Ejections (CME), i.e. expulsions of huge masses of plasma and magnetic field into the heliosphere, are often associated with soft X-ray (SXR) bursts (TandbergHanssen \& Emslie 1988), which are routinely observed by the GOES spacecraft. Statistical studies of correlations between the parameters of CMEs (such as the linear velocity) and the associated SXR bursts have led to conflicting results, ranging from no significant correlation (Aggarwal et al. 2008) to moderate correlations between SXR flux and CME speed (Moon et al. 2003, Vrsnak et al. 2005, Bein et al. 2012), or SXR flux and CME kinetic energy (Hundhausen, 1997, Burkepile et al. 2004). Since one can only measure the projected CME speed, an existing correlation may be smeared out if one considers CMEs irrespective of their location on the Sun. This can be one of the reasons for the low correlations shown in these studies. Moon et al. (2003), indeed, found a higher correlation when restricting their sample to limb events, and so did Burkepile et al. (2004), considering the kinetic energy of CMEs. In this work we investigate if the entire SOHO/LASCO data set since 1996 reveals a more significant correlation between CME speed and SXR peak flux near the limbs, where projection effects are minimized.

\section{Analysis and preliminary results}

This study is based on (1) a listing of the measured position angles (PA), widths, heights and speeds of CMEs provided in the LASCO/SOHO CME catalogue during the period 1996 - 2008; (2) the X-ray key parameters from GOES satellites obtained from the National Oceanic and Atmospheric Administration (NOAA) and National Geophysical Data Center (NGDC) during the same time period. We selected CMEs on the western $\left(\mathrm{PA}=60^{\circ}-120^{\circ}\right)$ and eastern $\left(\mathrm{PA}=240^{\circ}-300^{\circ}\right)$ solar limb with velocities $\geqslant 300 \mathrm{~km} / \mathrm{s}$. The associated SXR bursts were identified from the timing association and the linear speed of the CME. From this new list of CME/flare events, we selected those with heliolongitudes near the limb $\left(70^{\circ}-85^{\circ}\right.$ east and west) to evaluate the correlation between CME speed and SXR peak flux. A total of $77 \mathrm{CME}$ events fulfilled the selection criteria. 

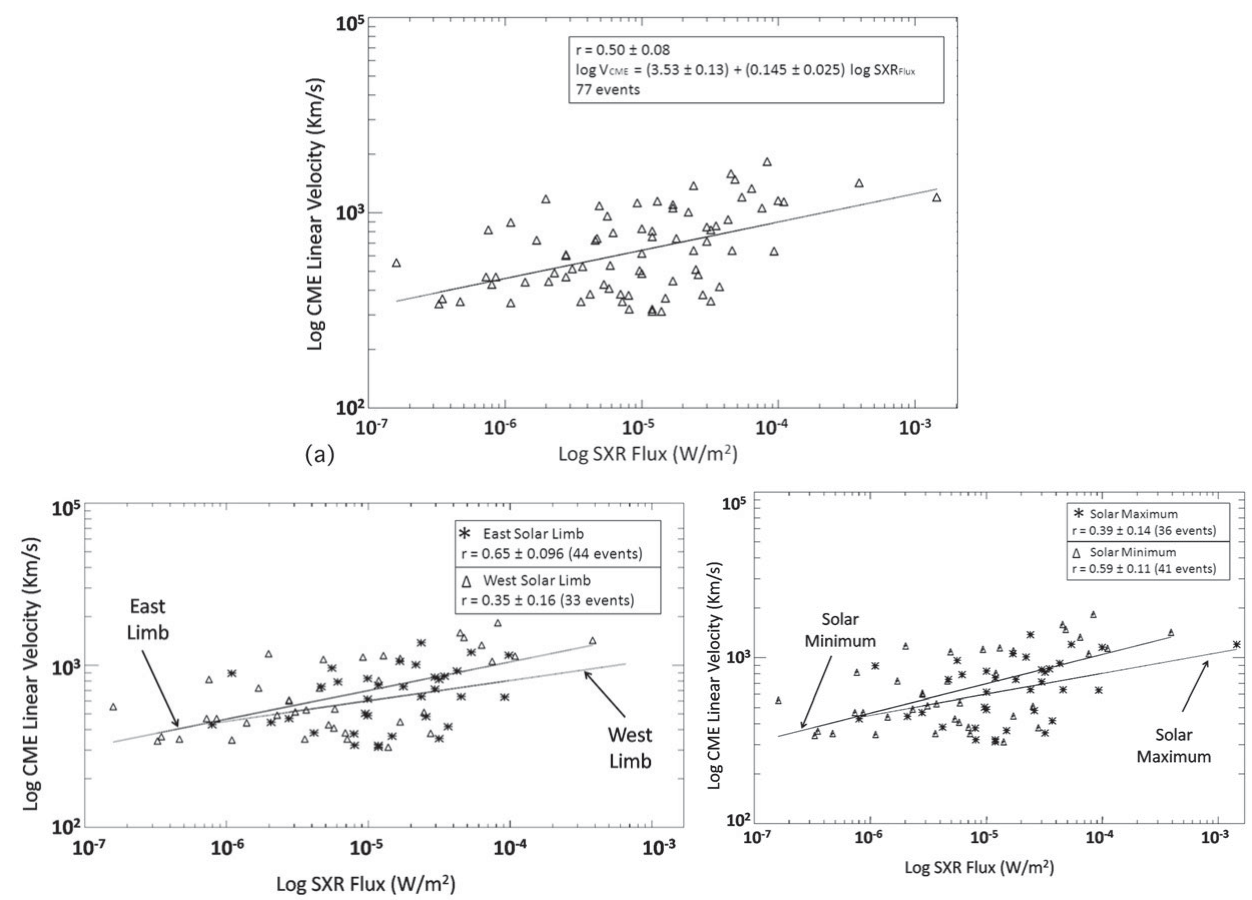

Figure 1. Logarithmic plot of CME velocity versus SXR peak flux of the associated flare for events originating near the eastern and western solar limb. (a) All events during the interval 1996-2008. (b) Separate plots for eastern and western events. (c) Separate plots for events near solar minimum and maximum.

We found a correlation of $r=0.50$ and a weak dependence (very similar to other authors) of the CME speed on SXR peak flux (Fig. 1a). The correlation is lower than found by Moon et al. (2003) for a small data set (24 events), but higher than that of Bein et al. (2012; $r=0.32$ for 70 events) who considered CMEs anywhere on the disk.

Higher correlations were found for (1) the east limb events (Fig. 1b) and (2) events around solar minimum as compared to solar maximum (Fig. 1c). It is presently not clear if result (1) is due to a selection effect or if it is physically relevant. Result (2) might be related to the clearer temporal separation of events in times of low solar activity.

\section{Acknowledgements}

C. S.-M. and L.T. acknowledge financial support for attending the symposium. We are grateful to the teams providing the LASCO CME and GOES X-ray catalogues.

\section{References}

Aggarwal, M., et al. 2008, J. Astrophys. Astr., 29, 195

Bein, B. M., et al. 2012, Astrophys. J., 755, 44

Burkepile, J. T., et al. 2004, J. Geophys. Res., 109, A03103

Hundhausen, A. J. 1997, in: J. R. Jokipii et al. (eds.), Cosmic Winds and the Heliosphere (Tucson: Univ. Arizona Press), p. 259

Moon, Y. J., et al. 2003, J. Korean Astron. Soc., 36, 61

Tandberg-Hanssen, E. \& Emslie, A. G. 1988, The Physics of Solar Flares (Cambridge: Cambridge Univ. Press)

Vrsnak, B., et al. 2005, Astron. Astrophys., 435, 1149 